\title{
Hyperbaric Oxygen Therapy in the treatment of Idiopathic Sudden Sensorineural Hearing Loss
}

\author{
Debasish Guha, ${ }^{1}$ Shaoni Sanyal, ${ }^{2}$ Chayan Bhattacharyya, ${ }^{3}$ Abhijit Santra,${ }^{4}$ Swagatam Banerjee ${ }^{2}$
}

\begin{abstract}
Backgrounds and objectives
$\underline{\text { ABSTRACT }}$

Idiopathic sudden sensorineural hearing loss (ISSNHL) is an entity whose diagnosis and treatment remain controversial to date. Various modalities of treatment have been tried with varying degrees of success. Hyperbaric oxygen therapy (HBOT) is a recent modality of treatment for this condition which acts by improving cochlear microcirculation. The objective of this study was to evaluate the effect of HBOT in improving hearing loss and secondary symptoms patients with ISSNHL.

Methods

This prospective study was undertaken over a 6 month period from the Department of Otorhinolaryngology in a tertiary referral hospital in eastern India. 48 consecutive newly diagnosed ISSNHL patients were included in this study. The patients were subjected to HBOT at 2 A.T.A (Atmosphere Absolute) for an hour over 45 days. Hearing status and secondary symptoms as assessed by clinical tests, pure tone audiogram were analysed before and after HBOT.

$\underline{\text { Results }}$

Males comprised $62.5 \%$ (30/48) of our study population, mean age of the population was $49.3 \pm 13.4$ years. 45/48 patients (93.7\%) had unilateral ISSNHL, while 3 (6.3\%) had bilateral ISSNHL. Average hearing loss in the affected ear before and after treatment was $79.96 \mathrm{dBHL}$ and $62.27 \mathrm{dBHL}$ respectively. The most common secondary clinical feature was tinnitus (27/48, $56.3 \%)$, followed by vertigo $(24 / 48,50 \%)$, aural fullness $(15 / 48,31.25 \%)$ and nystagmus $(5 / 48,10.41 \%)$. After treatment tinnitus and vertigo were showed marked improvement; (22/27, 81.48\%) and (18/24, 75\%). 5 out of 15 (33.34\%) patients showed reduced aural fullness. There was no improvement in patients suffering from nystagmus.

$\underline{\text { Keywords }}$

Hearing loss, Sensorineural; Hyperbaric oxygenation; Audiometry, Pure-Tone
\end{abstract}

I diopathic sudden sensorineural hearing loss (ISSNHL) was defined by Hughes (1996) as hearing loss of $30 \mathrm{~dB}$ HL in 3 or more contiguous frequencies in 72 hours or less. ${ }^{1}$ Incidence of ISSNHL has been reported to be about 8 per 100,000 person per year in a Taiwanese study. ${ }^{2}$ The hearing loss may occur in an ear which previously had normal hearing or in an ear with a pre-existing hearing loss.

Probable causes of sudden sensorineural hearing loss are autoimmune diseases like Cogan's syndrome, Behcet's disease, SLE; bacterial, viral and fungal infections; metabolic conditions such as diabetes mellitus and hypothyroidism; ototoxic drugs; trauma; neoplasms like vestibular schwannoma or cerebellopontine angle tumors and vascular causes which include cerebrovascular stroke, sickle cell disease, etc.
Hyperbaric oxygen therapy (HBOT) aims to address vascular insufficiency and improve the hearing loss in ISSNHL by improving cochlear microcirculation. The use of hyperbaric therapy dates back nearly 350 years. The first hyperbaric chamber was created in 1662 by Henshaw. The early chambers used compressed air instead of Oxygen. In 1917, two German inventors Bernhard and Heinrich Dräger applied pressurised

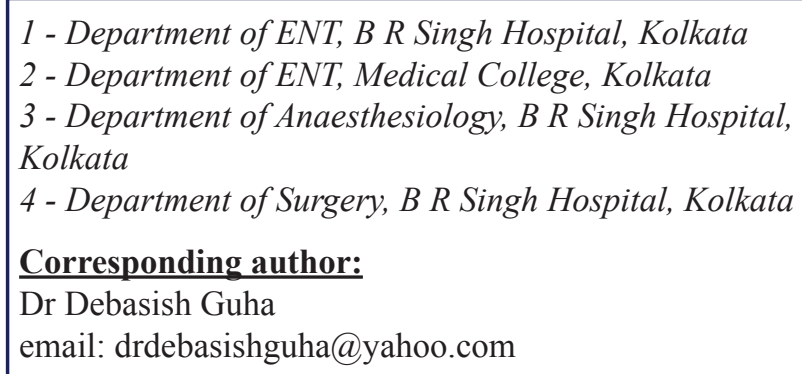


oxygen successfully in treating decompression illness from diving accidents. During the 1960s, studies began on a wide variety of indications for HBOT.

In a hyperbaric oxygen therapy chamber, the air pressure is increased to 2-3 times higher than normal air pressure. Under these conditions, more oxygen dissolves in the plasma. Oxygen dissolved in plasma has better bioavailability for the tissues because the increased concentration of oxygen proportionally increases the partial pressure of oxygen in arterial blood, and more oxygen can be delivered deeper into the tissue. ${ }^{3}$ The increased partial pressure of oxygen dissolved in the plasma compensates for hypoxia due to vascular insufficiency. HBOT also impacts the immune system. Oxygen has an antimicrobial effect, especially in anaerobic infections. HBOT has been shown to have beneficial effects on fibroblast activity and angiogenesis; to enhance the efficacy of leukocytes; to suppresses bacteria; to increase the efficacy of antibiotics; and to stimulate granulocytes' production of endogenous antimicrobial agents.

The aim of this study was to evaluate the effect of HBOT in improving hearing loss and secondary symptoms patients with ISSNHL.

\section{Materials and Methods}

The study was conducted between November, 2014 to April, 2015 in a tertiary care hospital in Eastern India, after obtaining clearance from the institutional Ethical Committee. All cases of sudden hearing loss underwent pure tone audiometry to ascertain the type of hearing loss. 124 cases were diagnosed to have sensorineural hearing loss (SNHL). Etiology of SNHL was determined by screening investigations which included complete haemogram, ESR, electrolyte profile, thyroid profile, lipid profile, fasting blood sugar, post prandial blood sugar, urea, creatinine, autoantibodies and MRI.

Out of 124 cases, 48 were included in the study as they met the following criteria of ISSNHL:

1. The hearing loss was sudden in onset (occurring within 72 hours) and sensorineural in nature as ascertained by Pure Tone Audiometry and

2. Hearing loss of $30 \mathrm{~dB}$ HL in $\geq 3$ contiguous frequencies and

3. No etiology could be determined even after screening investigations were carried out.

76 cases were excluded from the study because of one or more of the following reasons:

1. Poorly controlled blood sugar

2. Poorly controlled blood pressure

3. Seizures

4. Claustrophobia

5. Patients with pacemaker/ Arrhythmias

6. Patients on chemotherapy with doxorubicin / bleomycin / cisplatin

7. COPD

All patients were explained the details of the procedure and gave informed consent to undergo the procedure. The 48 patients that were included in the study were subjected to HBOT at 2 A.T.A for 1 hour in a monoplace chamber at another tertiary care centre (Fig. 1). The patient lay supine in the chamber and the chamber was then pressurised with 100\% oxygen. Pure tone audiometry was conducted at 15 days' intervals. The patients received HBOT for a duration of 45 days.

Assessment of hearing loss was recorded by pure tone audiometry. Pure tone average was taken at $500 \mathrm{~Hz}$, $1000 \mathrm{~Hz}, 2000 \mathrm{~Hz}$ and $4000 \mathrm{~Hz}$. Alteration of secondary symptoms was also recorded. The data was analysed to see if there was any significant improvement in hearing after 45 days of receiving HBOT.

\section{Results}

48 patients presented with sudden sensorineural hearing loss in whom no particular etiology could be determined and these patients were included in the study. Males comprised $62.5 \%(30 / 48)$ of our study population, mean age of the population was $(49.3 \pm 13.38) .45 / 48$ patients (93.7\%) had unilateral ISSNHL, while $3(6.3 \%)$ had bilateral ISSNHL. Average hearing loss in the affected ear before and after treatment was $79.96 \mathrm{~dB} \mathrm{HL}$ and $62.27 \mathrm{~dB}$ HL respectively. The cases of bilateral hearing loss were treated as individual cases, thus the number of ears affected with hearing loss was taken to be 51 . The 


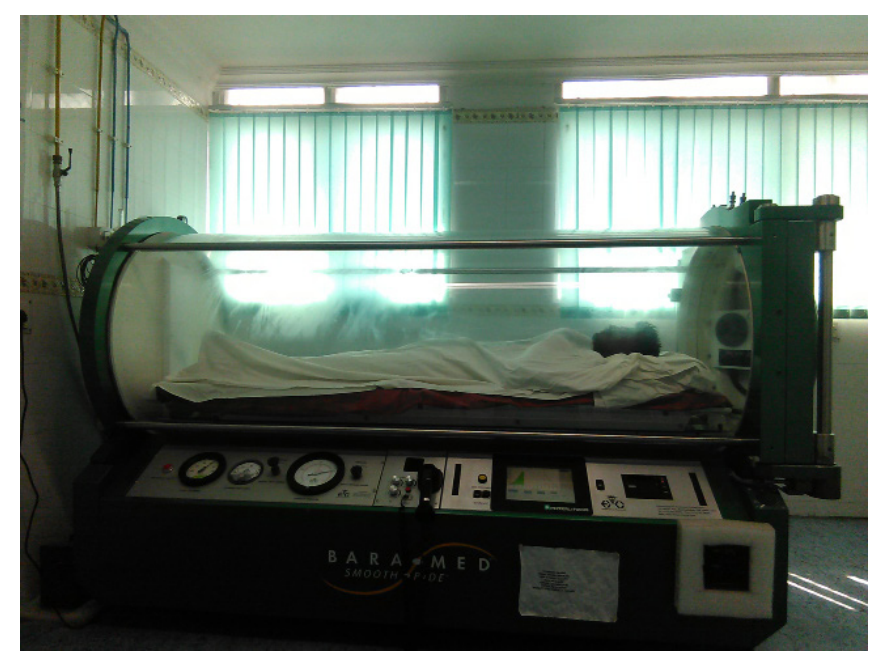

Fig. 1 Patient undergoing HBOT in monochamber

most common secondary clinical feature was tinnitus (27/48, 56.3\%), followed by vertigo (24/48, 50\%), aural fullness $(15 / 48,31.25 \%)$ and nystagmus (5/48, 10.41\%).

"Recovery" was defined as improvement of hearing loss $\geq 50 \%$ of pre-therapy hearing loss levels and resolution of secondary symptoms. (28/51, 54.90\%) cases with hearing loss showed recovery after HBOT.

"No recovery" was defined as improvement of hearing loss $\leq 50 \%$ of pre-therapy hearing loss levels and/or presence of secondary symptoms. $(12 / 51,23.52 \%)$ cases with hearing loss showed no recovery.

11 cases $(21.57 \%)$ showed a deterioration in hearing as compared to pre-procedural level of hearing loss. (Fig. 2)

Average hearing loss in the affected ear before treatment was 79.96dB HL and average hearing loss post treatment was $62.27 \mathrm{~dB}$ HL. Pure tone average in the non-affected ear before treatment was $26.75 \mathrm{~dB} \mathrm{HL}$ and pure tone average post treatment in the non-affected ear was 25.67dB HL. Students paired t-test (two tail) with unequal variance was performed on this data and it was seen that the difference in pure tone average before and after treatment hearing loss was significant $(p=0.0008)$. However, difference in pure tone average before and after treatment in the non-affected ear was not significant $(p=0.62)$. (Fig. 3$)$

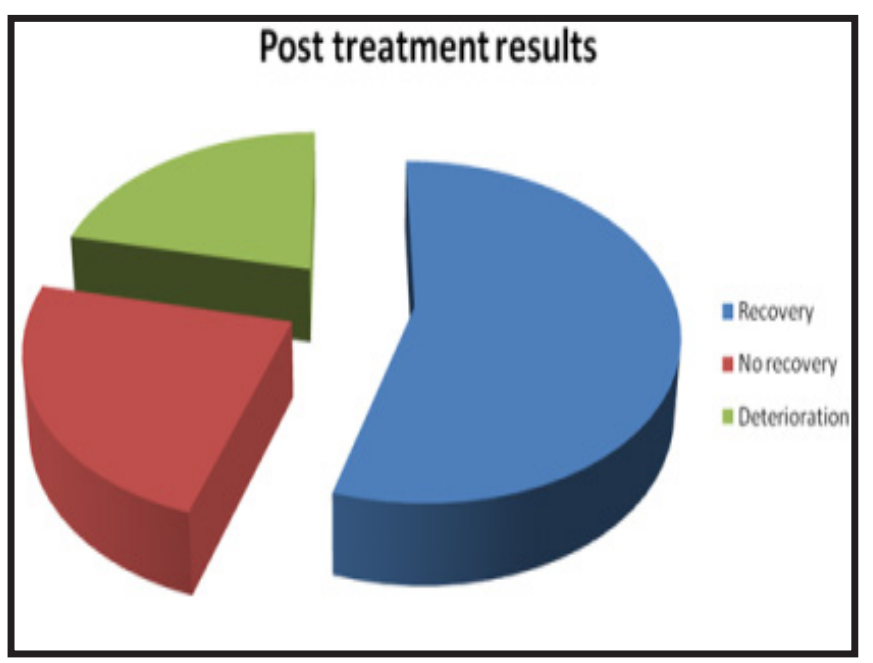

Fig. 2 Hearing Status after HBOT

\section{Discussion}

ISSNHL is a diagnosis of exclusion and due to a myriad of pathophysiological factors acting in this condition, different modalities of therapy have been advocated; yet there is no clear consensus regarding treatment protocol for ISSNHL. Systemic steroids, intratympanic steroids, ${ }^{4}$ antiviral medications ${ }^{5}$ and more recently HBOT $^{6,7,8,9,10,11}$ have been tried with varying degrees of success.

Many studies have tried to evaluate the effect of combining these therapies, $9,10,11,12,13$ and compared HBOT to the other forms of therapy. In these studies, there is significant improvement in hearing loss when HBOT is combined with the other conventional therapies.

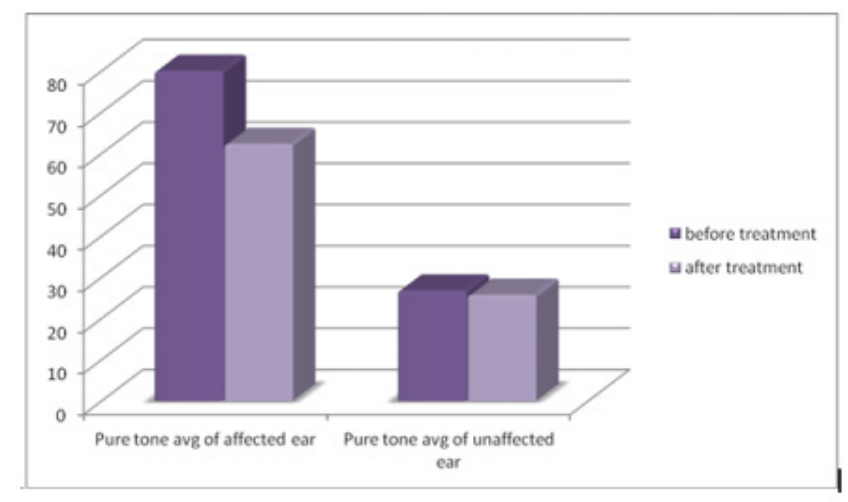

Fig. 3 Comparison of pre and post treatment pure tone averages in the affected and unaffected ears 
In a randomized control trial by Filipo $\mathrm{R}$ et al. comparing relative efficacy of HBOT vs Intratympanic steroid; 48 patients were studied, age varied from 37.6-54.5 years, and $56.25 \%$ were males. ${ }^{11}$ This is also in concordance with another retrospective study by Capuano et al. with 300 patients, age ranging from 52.24-54.05 years and 56\% were males. ${ }^{6}$ Our study is a prospective analytical study with 48 patients. The mean age of presentation in our study was 49.3 years and males comprised $62.5 \%$ of our patients.

In the study conducted by Capuano et al. 76/300 (25.34\%) had profound hearing loss; in the study designed by Filipo R et al., 25/48 (52.08\%) patients presented with severe hearing loss and 23/48 (47.91\%) had profound hearing loss. ${ }^{6,11}$ In our study, the average hearing loss was $79.96 \mathrm{~dB}$ HL.

Capuano et al. ${ }^{6}$ divided 300 patients into 3 groups each with 100 patients - Group A (Intravenous steroid(IVS)), Group B (HBOT), and Group C (IVS + HBOT). Intravenous steroid (methylprednisolone) was given in decreasing doses of $40 \mathrm{mg}$ for 7 days, and then $20 \mathrm{mg}$ for 3 days. 16 HBOT sessions were given at 2.5 A.T.A for 90 minutes.

Results were classified as follows:

- Good recovery- hearing within $15 \mathrm{~dB}$ of unaffected ear

- Fair recovery- Hearing improvement $>10 \mathrm{~dB}$ but not within $15 \mathrm{~dB}$ of unaffected ear.

- Poor recovery- hearing level with no change, or improvement $<10 \mathrm{~dB}$, or deterioration

Good recovery was seen in $84 \%$ in Group C (IVS + HBOT), $70 \%$ in Group B (HBOT) and 68\% in Group A (IVS). ${ }^{6}$

In the study conducted by Filipo $\mathrm{R}$ et al, ${ }^{11}$ patients were divided into 2 groups. 25 patients were in the group with severe hearing loss (70-90dB) and the rest were in the group with profound hearing loss ( $>90 \mathrm{~dB})$. Patients in each group were randomised into two streams - those that received Intratympanic steroids (ITS) + HBOT and those that received Intravenous steroids (IVS) and HBOT. HBOT was given for ten sessions at 2.4 A.T.A for 75 minutes. Complete recovery was defined as PTA $\leq 25 \mathrm{~dB}$ HL or identical to contralateral non-affected ear or PTA improvement $>30 \mathrm{~dB}$. (Table I)

Table I: Complete recovery in study by Filipo $\mathrm{R}$ et al. ${ }^{11}$

\begin{tabular}{|c|c|c|}
\hline $\begin{array}{c}\text { COMPLETE } \\
\text { RECOVERY }\end{array}$ & $\begin{array}{c}\text { SEVERE } \\
\text { ISSNHL }\end{array}$ & $\begin{array}{c}\text { PROFOUND } \\
\text { ISSNHL }\end{array}$ \\
\hline ITS +HBOT & $83.3 \%$ & $60 \%$ \\
\hline IVS + HBOT & $53.8 \%$ & $53.8 \%$ \\
\hline
\end{tabular}

In our study, HBOT was given at 2A.T.A for 60 minutes for 45 days. Recovery was defined as improvement of hearing loss $\geq 50 \%$ of pre-therapy hearing loss levels and resolution of secondary clinical features. 28/51 $(54.90 \%)$ cases with hearing loss showed recovery.

No Recovery was defined as improvement of hearing loss $\leq 50 \%$ of pre-therapy hearing loss levels and/or presence of secondary clinical features. $12 / 51(23.52 \%)$ cases with hearing loss showed no recovery.

11 cases $(21.57 \%)$ showed a deterioration in hearing as compared to pre-procedural level of hearing loss.

Our study also evaluated the effect of HBOT on secondary clinical features. After treatment, patients with tinnitus and vertigo showed marked improvement - 22/27 (81.48\%) and 18/24 (75\%) respectively. 5/15 (33.34\%) of patients showed reduced aural fullness. There was no improvement in patients suffering from nystagmus. (Fig.4)

To the best of our knowledge there has been no study in which the effect of HBOT alone has been studied. In our study, we have seen a significant improvement in hearing with HBOT alone.

\section{Conclusion}

HBOT is a safe and emerging therapy in treating ISSNHL. Till date it has been used as an adjunct to other therapeutic modalities but shows promise to be efficacious independently. However, larger trials are required before HBOT can be incorporated into routine clinical practice. 


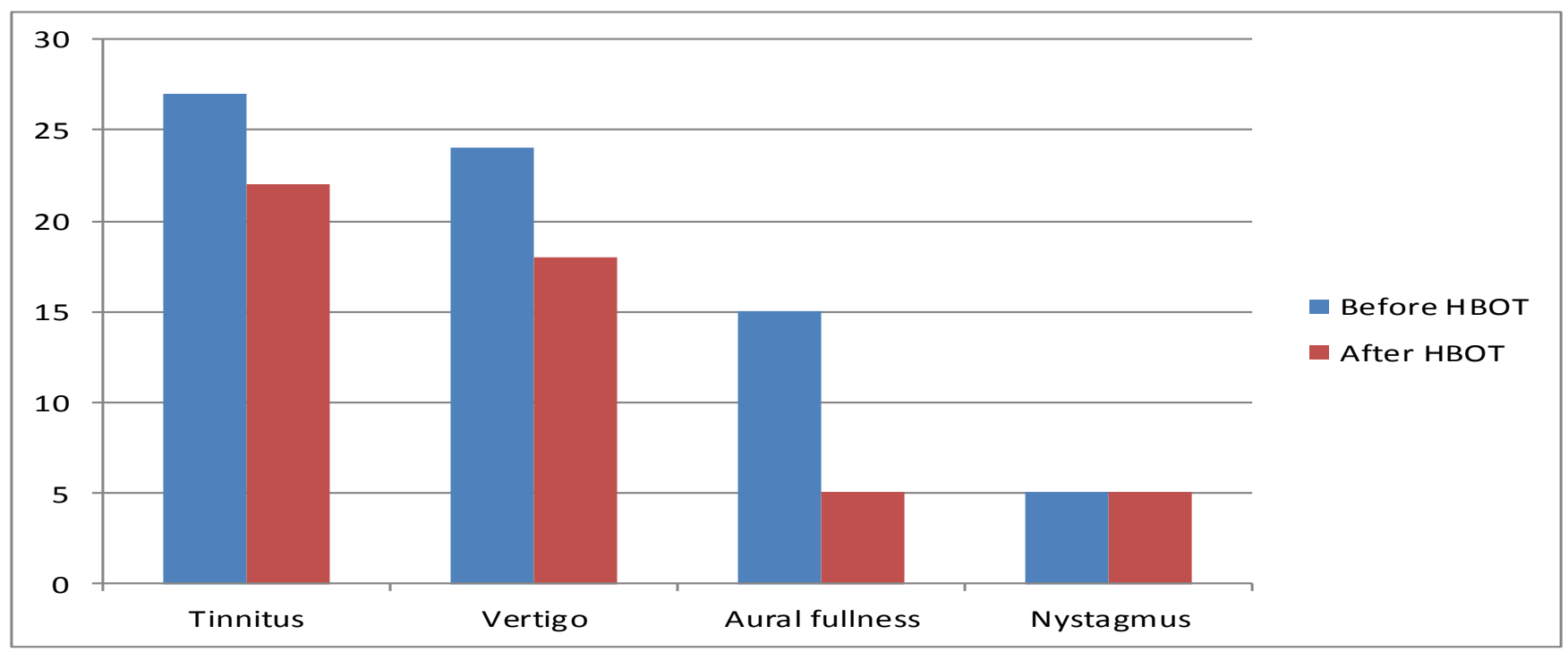

Fig. 4 Effect of HBOT on secondary clinical features

\section{References}

1. Wilson WR, Byl FM, Laird N. The efficacy of steroids in the treatment of idiopathic sudden hearing loss. A doubleblind clinical study. Archives of Otolaryngology 1980; 106: 772-6

2. Wu CS, Lin HC, Chao PZ. Sudden sensorineural hearing loss: Evidence from Taiwan. Audiology and Neurootology 2006; 11:151-6

3. Tompach PC, Lew D, Stoll JL. Cell response to hyperbaric oxygen treatment. Int J Oral Maxillofac Surg. 1997;26(2):8286.

4. Crane RA, Camilon M, Ngyuen S, Meyer TA. Steroids for Treatment of Sudden Sensorineural Hearing Loss: A MetaAnalysis of Randomized Controlled Trials.Laryngoscope 2015; 125(1):209-17.

5. Shaikh JA, Roehm PC. Does addition of antiviral medication to high-dose corticosteroid therapy improve hearing recovery following idiopathic sudden sensorineural hearing loss? Laryngoscope 2011;121(11):2280.

6. Capuano L, Cavaliere M, Parente G, Damiano A, Pezzuti G, Lopardo D, Iemma M. Hyperbaric oxygen for idiopathic sudden hearing loss:Is the routine application helpful? Acta Otolaryngol. 2015; 135(7):692-7.

7. Yacono D, Allen MW. Hyperbaric oxygen therapy (HBO2) as an adjunct in the treatment of idiopathic sudden sensorineural hearing loss(ISSHL)--A case report on two patients. Undersea Hyperb Med. 2014; 41(5):401-5.

8. Gaitanou K, Fildissis G, Vavasis P, Kalentzos V, Baltopoulos G. Management of sudden hearing loss with hyperbaric oxygen therapy. Undersea Hyperb Med. 2014; 41(5):363-70.

9. Cvorovic L, Jovanovic MB, Milutinovic Z, Arsovic N, Djeric D. Randomized prospective trial of hyperbaric oxygen therapy and intratympanic steroid injection as salvage treatment of sudden sensorineural hearing loss. Otol Neurotol. 2013; 34(6):1021-6.

10. Bennett $\mathrm{MH}$, Kertesz $\mathrm{T}$, Perleth $\mathrm{M}$, Yeung P, Lehm JP. Hyperbaric oxygen for idiopathic sudden sensorineural hearing loss and tinnitus. Cochrane Database Syst Rev. 2012; 17:10:CD004739

11. Filipo R, Attanasio G, Viccaro M, Russo FY, et al. Hyperbaric oxygen therapy with short duration intratympanic steroid therapy for sudden hearing loss. Acta Otolaryngol.2012; 132(5):475-81.doi 10.3109/00016489.2011.647360

12. Holy R, Navara M, Dosel P, Fundova P, Prazenica P, Hahn A. Hyperbaric oxygen therapy in idiopathic sudden sensorineural hearing loss (ISSNHL) in association with combined treatment. Undersea Hyperb Med. 2011; 38(2):137-42

13. Alimoglu Y, Inci E, Edizer DT, Ozdilek A, Aslan M. Efficacy comparison of oral steroid, intratympanic steroid, hyperbaric oxygen and oral steroid + hyperbaricoxygen treatments in idiopathic sudden sensorineural hearing loss cases. Eur Arch Otorhinolaryngol. 2011;268(12):1735-41. doi: 10.1007/ s00405-011-1563-5 